\title{
University remote teaching in crystal chemistry during the COVID-19 pandemic: positive and negative aspects
}

\author{
O. V. Grineva \\ Chemistry Department of Moscow M. V. Lomonosov State University, Moscow, Russia \\ ovg@phys.chem.msu.ru
}

At the Chemistry Department of the Moscow M. V. Lomonosov State University, the compulsory course "Crystal Chemistry" and numerous elective courses for undergraduate and postgraduate students (devoted to the in-depth study of several crystallographic and crystallochemical topics) do not include extensive practical classes that can be performed only in specially equipped rooms. Therefore, in principle, they can be done remotely without significant changes in content. In fact, remote learning at the Department in the last three semesters differs significantly from the option of education that was called distance learning before the COVID-19 pandemic. Classical distance learning assumes that the student can become acquainted with the teaching materials and perform test tasks conveniently within a specified period. The current procedure of remote learning at the Department may be called classroom-like: there is a schedule of lectures and seminars, while teachers are obliged to conduct live seminars, and live lectures are highly desirable.

Although before the pandemic, in most of the lectures and seminars on the general course "Crystal Chemistry", the teachers demonstrated space models (polyhedra, individual molecules, Bravais lattices, models demonstrating the effect of symmetry elements, unit cells of inorganic and organic substances), and for some students, this facilitated the perception of the material, but strictly speaking, such demonstrations are not mandatory for a successful understanding of the course. Since 2009, the course includes acquaintance with structure visualization programs, and students have access to cif files for the structures on the must-have list. Before the pandemic, work with such programs was mainly done in the classroom, but students were recommended to download demo versions of the Diamond and Mercury programs for deeper insight, so the teachers already had some experience, which is difficult for students. During the "pandemic" semesters, the work with these programs was done completely at home, and most of the students coped with it successfully. Unfortunately, independent searches according to different criteria in the CSD, which were previously done by students of one of the specialized groups, were not implemented (due to licensing restrictions and a very large database). Demonstration of searches from the teacher's computer were not quite complete in Zoom when using the usual option of screen sharing since inner windows in ConQuest were not visible, and the setting in Zoom allowing to see everything was undesirable when working with students. Therefore, it was necessary to make additional slides for the presentations. Nevertheless, because the use of computer programs became part of the "Crystal Chemistry" course long before the pandemic, difficulties in the transformations to remote learning were mainly due to the suddenness of the jump and technical problems among some students and teachers (the lack of devices allowing to set up necessary programs and to work with them, as well as with remote resources, poor Internet connection).

The advantages of the remote format for students include watching videos (teachers were required to record both lectures and seminars) as many times as they need to understand. In addition to the videos, many teachers shared other materials. Unfortunately, this format fostered complacency for some students, but overall, the students' responses after completing the courses were not worse than in the face-to-face studies.

For teachers, the distance format makes it generally easier to carry out current control of knowledge using computer tests (Moodle is the main program at the Department for these purposes), although, of course, preparing good tests takes a lot of time. Unfortunately, computer tests cannot adequately assess students' knowledge for all sections of the "Crystal chemistry" course, and in those cases, when students placed the answers to the tasks in the form of pictures, their verification took significantly longer than checking traditional paper forms.

For me, the most inconvenient and time-consuming part of fully remote learning compared to the face-to-face format was oral exams using Zoom and MS Teams. This is partly due to imperfect programs (for example, in MS Teams, it is inconvenient to draw, much more often than in Zoom, there was the poor quality of communication), but more importantly, it is impossible to determine with absolute certainty whether the student really has a bad connection or is deliberately wasting time and creating interference to somehow find the answer to the question asked.

Keywords: distance education; Zoom; Moodle; Diamond; Mercury 